ORIGINAL ARTICLE

\title{
Accuracy of the volume and pressure displays of high frequency oscillators
}

\author{
J A Leipälä, S Iwasaki, A Milner, A Greenough
}

Arch Dis Child Fetal Neonatal Ed 2004;89:F174-F176. doi: 10.1136/adc.2002.023937

See end of article for authors' affiliations

.....................

Correspondence to: Professor Greenough, Department of Child Health, King's College Hospital, London SE5 9PJ, UK; anne.greenough@ kcl.ac.uk

Accepted 11 March 2003
Objective: To determine the effect of frequency on the accuracy of volume and pressure displays of high frequency oscillators.

Methods: The effect of frequency on the displayed volume of the Stephanie, Dräger Babylog 8000 Plus, and SLE 5000 oscillators was assessed. A sine wave pump delivered a constant tidal volume at frequencies of $5-15 \mathrm{~Hz}$ to the patient manifold of the oscillators. The displayed volumes at each frequency were compared with the delivered volume. The effect of frequency on displayed pressure was assessed by connecting the oscillator's patient manifold to a lung model; three types of oscillator were studied (SensorMedics 3100A, SLE 5000, and Stephanie). Airway pressure was measured from the manifold using a pressure transducer and non-compliant tubing; the pressure measuring system had a flat frequency response to $30 \mathrm{~Hz}$.

Results: The SLE 5000 volume display overread the delivered volume (by about $5 \%$ ), but was not affected by frequency. At $5 \mathrm{~Hz}$, the Dräger Babylog 8000 Plus and the Stephanie underread the delivered volume (by about 20\%). Increasing frequency resulted in a greater discrepancy between the delivered and displayed volume with the Stephanie, but a smaller discrepancy with the Dräger Babylog 8000 Plus. Altering frequency had a small effect (maximum difference 6\%) on the relation between the displayed and delivered pressure for all three oscillators.

Conclusion: Frequency affects the accuracy of displayed volumes and, to a lesser extent, displayed pressures of high frequency oscillators. The results emphasise that data displayed by new devices should not be uncritically accepted.
$\mathrm{H}$ igh frequency oscillation (HFO) is now commonly used in neonatal practice, both to try to "rescue" infants with severe respiratory failure ${ }^{1}$ and "prophylactically" with the aim of reducing chronic lung disease. ${ }^{2}$ Several types of oscillator are available; in a recently published randomised trial, ${ }^{3}$ three types of oscillator were used. In such trials and clinical practice, serial recordings are often made of the displayed pressure and used to indicate disease severity and document changes in the infant's condition. The displayed pressure and volume are also used to guide the settings chosen when infants are transferred from one oscillator type to another. It is essential therefore that the displayed values are accurate. Unfortunately, it has been shown that the relation between the displayed and delivered pressure differs between oscillators: some overestimate whereas others underestimate the delivered pressure. ${ }^{4}$ A variety of frequencies are used during HFO, usually between 10 and $15 \mathrm{~Hz}$ in neonatal patients, but, if there is carbon dioxide retention despite use of high oscillatory amplitudes, then frequency is reduced below $10 \mathrm{~Hz}$. Oscillator performance is influenced by frequency, as indicated by the inverse relation between frequency and delivered volume. ${ }^{56}$ The influence of compliance on the oscillatory pressure waveform at the airway opening and the delivered volume has been shown to be also dependent on the frequency and specific oscillator used. ${ }^{7}$ It is therefore important to determine the accuracy at different frequencies of the pressure and volume displays of high frequency oscillators in current use and this is the aim of this study.

\section{METHODS}

\section{Accuracy of volume display}

The accuracy of the volume displays of the Stephanie (Stephan Biomedical Incorporation, Gackenbach, Germany), the Dräger Babylog 8000 Plus (Dräger Medizintechnik GmbH, Germany), and the SLE 5000 (Specialised Laboratory Equipment Ltd, South Croydon, UK) oscillators was examined in vitro. In the Stephanie oscillator, the waveform is generated by movements of a piston mounted in the inspiratory/expiratory block, exposing the infant to positive and negative pressures. In the SLE 5000, oscillation is generated through a forward and a reverse jet both controlled by high speed valves. The mechanisms of the other oscillators tested have been described elsewhere in detail. ${ }^{6}$ A constant tidal volume $(4 \mathrm{ml})$ was delivered to the patient manifold using a variable speed, sine wave pump. ${ }^{8}$ This was achieved by connecting the inspiratory connection of the patient manifold to the output of the pump by a $10 \mathrm{~cm}$ rigid plastic tube and occluding the expiratory connection of the manifold. The patient connector of the manifold was attached to a $3 \mathrm{~mm}$ endotracheal tube with the distal end open to the atmosphere. The output of the sine wave pump was constant over the frequency range $(5-15 \mathrm{~Hz})$ studied. The effect of frequencies from 5 to $15 \mathrm{~Hz}$ was examined; frequency was increased in $1 \mathrm{~Hz}$ increments. At each frequency, the displayed volume was recorded and expressed as a percentage of the $4 \mathrm{ml}$ delivered by the sine wave pump.

\section{Accuracy of pressure display}

The effect of frequency on the displayed pressure of the SensorMedics 3100A (SensorMedics Corporation, Anaheim, California, USA), the SLE 5000 (Specialised Laboratory Equipment Ltd), and the Stephanie (Stephan Biomedical Incorporation) oscillators was assessed. The ventilators were connected to a lung model, which had a compliance of $0.8 \mathrm{ml} / \mathrm{cm} \mathrm{H}_{2} \mathrm{O}$ and a resistance of $70 \mathrm{~cm} \mathrm{H}_{2} \mathrm{O} / \mathrm{l} / \mathrm{s}$ at a flow rate of 2 litres/min. The model consisted of an endotracheal tube $(3 \mathrm{~mm})$ sealed in a rubber bung, which was inserted 
into the open neck of a 1 litre conical flask. To assess the effect of frequency on the accuracy of the displayed oscillatory pressure, a reference pressure measuring system was used. The reference pressure measuring system consisted of a pressure transducer (Validyne MP 45, range $\pm 50 \mathrm{~cm} \mathrm{H} \mathrm{H}_{2} \mathrm{O}$; Validyne Corporation, Northridge, California, USA) and tubing attached to the oscillator manifold at the site of the oscillator's pressure measuring line. To avoid oscillations in the reference pressure measuring line, short $(7 \mathrm{~cm})$ non-compliant tubing with an internal diameter of $4 \mathrm{~mm}$ was used. The frequency response of the reference pressure measuring system was flat to $30 \mathrm{~Hz}{ }^{7}$ The output of the reference pressure transducer was recorded on a storage oscilloscope with printout facility. The ventilator's displayed pressure was related to the deflection on the storage oscilloscope at each frequency, and the resultant ratio expressed as a percentage of the ratio at $5 \mathrm{~Hz} ; 5 \mathrm{~Hz}$ was selected for baseline comparison as this is the highest frequency at which the standard volume measuring device, the pneumotachograph, performs reliably. Throughout the range of frequencies examined, the inspiratory to expiratory ratio was kept constant at $1: 1$, and the mean airway pressure at $12 \mathrm{~cm} \mathrm{H} \mathrm{H}_{2} \mathrm{O}$. At $5 \mathrm{~Hz}$, the oscillatory amplitude was set at $50 \mathrm{~cm} \mathrm{H}_{2} \mathrm{O}$, and the setting then remained unchanged throughout the range of frequencies.

\section{RESULTS}

At $5 \mathrm{~Hz}$, there was a discrepancy between the measured and displayed volume for all of the oscillators examined (fig 1); this was smallest for the SLE 5000. Both the Dräger Babylog 8000 Plus and Stephanie underrecorded the volume at $5 \mathrm{~Hz}$ by about $20 \%$, but the effect of increasing frequency on the discrepancy between the measured and displayed volumes differed between the two oscillator types (fig 1). Increasing frequency had a variable effect on the accuracy of the displayed pressure of the Stephanie oscillator. As frequency was increased, the display of the SensorMedics and SLE 5000 overrecorded the displayed pressure, but the effect was small (maximum difference 6\%; fig 2 ).

\section{DISCUSSION}

We have shown that the accuracy of the volume display of the oscillators studied differed as frequency was increased. The greatest discrepancy between the displayed and delivered volume was seen with the Stephanie oscillator, which incorporates a pneumotachograph to measure flow.

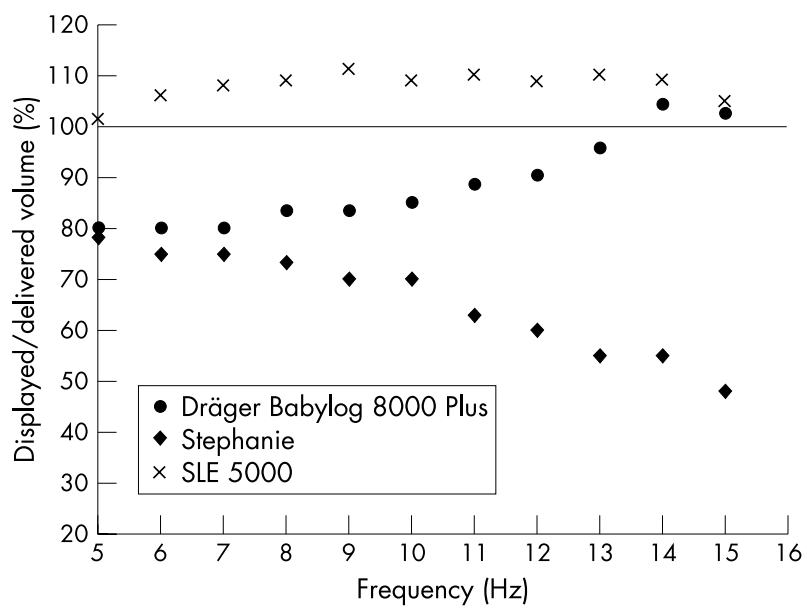

Figure 1 Effect of frequency on the accuracy of the volume display.

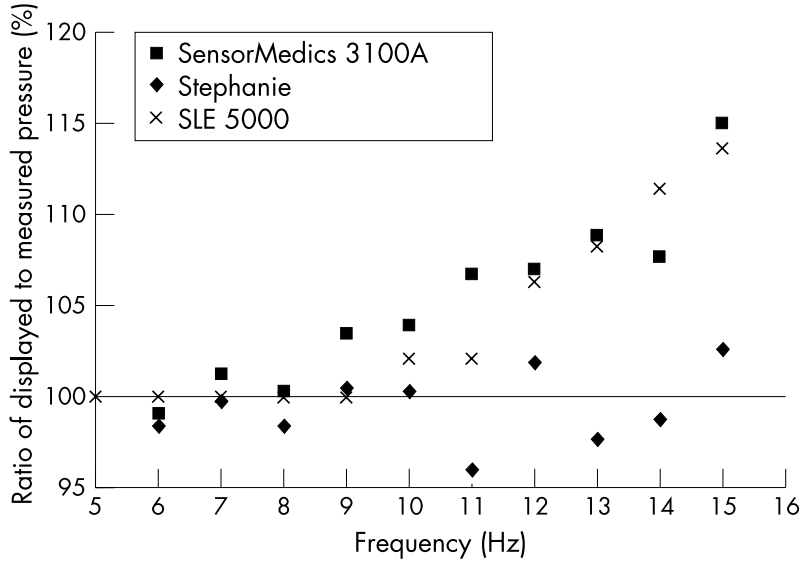

Figure 2 Effect of frequency on the accuracy of the displayed pressure.

Delivered volume during HFO has been measured in research studies using a pneumotachograph. ${ }^{9}{ }^{10}$ The accuracy of such a system has been compared with simultaneous measurements by plethysmography, ${ }^{11}$ and a good correlation $(r=0.92)$ was found. That correlation, however, required that the system had a suitable frequency of response. Unless that is taken into account, pneumotachographs may underrecord at high frequencies. ${ }^{12}$ The Dräger Babylog 8000 Plus and SLE 5000 oscillators use thermistors to measure flow. A hot wire anemometer (thermistor) monitoring system has been shown to provide reliable measurements of tidal volume at the airway opening. ${ }^{13}$ Our results suggest that during HFO, volume measurement using thermistors is more accurate than the use of a pneumotachograph, as there was greater linearity of response across the range of frequencies tested. ${ }^{12}$

Differences in the accuracy of the pressure display with increasing frequency were also found, although the effect was relatively small (fig 2). The displayed pressures were compared with the recordings from the reference pressure measuring system. We do not feel that the differences in the performance of the pressure measuring system with increased frequency accounted for the results, as we have previously shown that the system had a flat frequency response up to $30 \mathrm{~Hz}^{8}$ In addition, all three oscillators were compared with the same reference system, and yet differences in the accuracy of their displays were seen as frequency was increased. A possible explanation for the differences seen is that, as frequency was increased, oscillations may have been set up in the tubing incorporated into the oscillator's measuring system, which ran from the manifold to the pressure transducers within the oscillator. Standard circuits were used with all three oscillators, which incorporated relatively long tubing but, between the oscillator types, the length and diameter varied, which would influence pressure waveform damping and hence may explain the differences in the accuracy of their pressure displays seen with increasing frequency. Alternative explanations are differences in impedance characteristics of the pressure transducer tubing from the patient manifold to the oscillator pressure transducer or differences in the characteristics of the pressure transducer incorporated into the oscillator.

For consistency, an inspiratory to expiratory ratio of $1: 1$ was used with all of the oscillators. The inspiratory to expiratory ratio has been shown to influence the intratracheal and intra-alveolar pressure during HFO. ${ }^{14-16}$ The SensorMedics can be used with an inspiratory to expiratory ratio of $1: 1$ or $1: 2$. It has, however, been previously shown that, at an inspiratory to expiratory ratio of $1: 2$, the mean 
alveolar pressure is lower than the displayed mean airway pressure, whereas at a ratio of 1:1 the displayed and actual pressures are about equal. ${ }^{4}$ It has been suggested that at a $1: 1$ ratio, there will be a similar relation between the displayed and actual pressures with other sine wave generators. ${ }^{4}$ The Dräger Babylog 8000 Plus, however, has been shown to display lower pressures than the intrapulmonary pressures. This means that, if there is a switch from a SensorMedics 3100A to a Dräger Babylog 8000 Plus oscillator at a displayed pressure of $12 \mathrm{~cm} \mathrm{H}_{2} \mathrm{O}$, this could result in a $5-8 \mathrm{~cm} \mathrm{H}_{2} \mathrm{O}$ abrupt increase in the actual distending pressure. ${ }^{4}$ Similar differences in the relation between the displayed oscillatory amplitude pressure and the transpulmonary pressure swings may occur between the oscillators. This would mean that the differences we found in the accuracy of the displayed pressure between oscillators with increasing frequency would further compound the problems encountered on transferring from one oscillator to another.

In conclusion, at the frequencies used in clinical practice, the accuracy of the volume and pressure displays of neonatal oscillators varies as frequency is increased. These results emphasise that data displayed by new devices cannot be accepted uncritically and that results from different devices cannot be considered interchangeable. It cannot be assumed that, if an infant is transferred from one oscillator type to another at the same settings, the same volumes and pressures will be delivered. We would therefore recommend that careful assessment of chest vibration is made throughout, and the infant's blood gases are checked shortly after such a transfer has taken place, so that, if necessary, appropriate changes in settings are rapidly made to return the infant's blood gases to the predetermined range. Further development of accurate and frequency independent volume monitoring techniques is essential to optimise use of HFO ventilation.

\section{ACKNOWLEDGEMENTS}

JAL is supported by the Finnish Pediatric Research Foundation and the Finnish Medical Foundation, and SI by the King's College Hospital/SLE Ltd Research Fellowship. We thank Ms Sue Williams for secretarial assistance.

\section{Authors' affiliations}

J A Leipälä, S Iwasaki, A Milner, A Greenough, Children Nationwide Neonatal Intensive Care Unit, King's College Hospital, London, UK

\section{REFERENCES}

1 Bhuta T, Clark RH, Henderson-Smart DJ. Rescue high frequency oscillatory ventilation vs conventional ventilation for infants with severe pulmonary dysfunction born at or near term. (Cochrane Review). Cochrane Library. Issue 4. Oxford: Update Software, 2001.

2 Henderson-Smart DJ, Bhuta T, Cools F, et al. Elective high frequency oscillatory ventilation versus conventional ventilation for acute pulmonary dysfunction in preterm infants. (Cochrane review). Cochrane Library. Issue 3. Oxford: Update Software, 2001.

3 Johnson AH, Peacock JL, Greenough A, et al. High-frequency oscillatory ventilation for the prevention of chronic lung disease of prematurity. N Engl J Med 2002;347:633-42.

4 Hatcher D, Watanabe H, Ashbury T, et al. Mechanical performance of clinically available, neonatal, high-frequency, oscillatory-type ventilators. Crit Care Med 1998;26:1081-8

5 Fredberg JJ, Glass GM, Boynton BR. Factors influencing mechanical performance of neonatal high-frequency ventilators. J Appl Physiol 1987;62:2485-90.

6 Laubscher B, Greenough A, Costeloe K. Performance of four neonatal high frequency oscillators. British Journal of Intensive Care 1996;6:148-52.

7 Pillow JJ, Wilkinson MH, Neil HL, et al. In vitro performance characteristics of high-frequency oscillatory ventilators. Am J Respir Crit Care Med 2001;164:1019-24.

8 Hoskyns EW, Milner AD, Hopkin IE. Measurement of tidal lung volumes in neonates during high-frequency oscillation. J Biomed Eng 1992;14:16-20.

9 Boynton BR, Hammond MD, Fredberg JJ, et al. Gas exchange in healthy rabbits during high frequency oscillatory ventilation. J Appl Physiol 1989;66:1343-51.

10 Watson JW, Jackson AC. $\mathrm{CO}_{2}$ elimination as a function of frequency and tidal volume in rabbits during HFO. J Appl Physiol 1984;57:354-9.

11 Courtney SE, Weber KR, Spohn WA, et al. Measurement of tidal volume using a pneumotachometer during high-frequency oscillation. Crit Care Med 1990;18:651-3.

12 Chan V, Greenough A, Milner AD. The effect of frequency and mean airway pressure on volume delivery during high-frequency oscillation. Pediatr Pulmonol 1993;15:183-6.

13 Scalfaro P, Pillow JJ, Sly PD, et al. Reliable tidal volume estimates at the airway opening with an infant monitor during high-frequency oscillatory ventilation. Crit Care Med 2001;29:1925-30.

14 Thome U, Pohlandt F. Effect of the TI/TE ratio on mean intratracheal pressure in high-frequency oscillatory ventilation J Appl Physiol 1998;84:1520-7.

15 Pillow JJ, Neil H, Wilkinson $\mathrm{MH}$, et al. Effect of $\mathrm{I} / \mathrm{E}$ ratio on mean alveolar pressure during high-frequency oscillatory ventilation. J Appl Physiol 1999;87:407-14.

16 Pillow JJ, Sly PD, Hantos Z, et al. Dependence of intrapulmonary pressure amplitudes on respiratory mechanics during high-frequency oscillatory ventilation in preterm lambs. Pediatr Res 2002;52:538-44. 